\title{
Idiopathic Hypoparathyroidism Presenting as Adult Onset Seizures
}

\author{
Ajaz Nabi Koul, M.D., Shamim Ahmad Bhat, M.D., Ridwana Ahad, M.B. \\ Pediatrics and Medicine, Sher-i-Kashmir Institute of Medical Sciences, Soura, Srinagar, Kashmir.
}

\section{A B S T R A C T}

Idiopathic hypoparathyroidism is a rare disorder of unknown etiology. It usually presents by second decade of life but delayed manifestations are known to occur. It presents as neuromuscular excitability, numbness, carpopedal spasm, cramps, laryngeal stridor and convulsions.

In subtle cases of hypoparathroidism neuromuscular excitability can be made evident by eliciting Chovstek's and Trousseu's sign. Typical ECG manifestations are QTC prolongation. Cataract, intracranial calcification are well known entities. ${ }^{2}$

Various other manifestations are Parkinsonism, Choreo athetosis, Spasm etc. ${ }^{3}$ Typically patients have low serum calcium, high inorganic phosphorus with low to undetectable PTH. In all such patients renal failure, chronic Gl disorders, persistent alkalosis, rickets and osteomalacia should be excluded. JMS 2012;15(2):182-83

Key words: Hypoparathyroidism, seizure

Idiopathic hypoparathyroidism is an uncommon condition characterized by the absence, fatty replacement or atrophy of the parathyroid glands. It may be familial or sporadic.

\section{Case}

Our patient M.I, 44 year old Saudi male non diabetic, non hypertensive having BMI of 24 presented with generalized tonic clonic seizures of two days duration. Historically patient had similar episodes eight and four months back prior to admission at our hospital for which he sought no medical advice.

Clinical examination revealed conscious, cooperative, well oriented male with normal vitals. Chest, CVS and

\section{Correspondence:}

Ajaz Nabi Koul MD, MRCP(UK), FCAN(Neurology)

Assistant professor medicine

Sher Kashmir Institute of Medicial sciences, Srinagar, 190011 ,

Kashmir, india

E-mail: ajazkoul@yahoo.com abdominal examination was unremarkable. CNS examination revealed normal higher mental functions, normal cranial nerves, normal motor and sensory examination. Fundoscopy was normal.

Complete blood counts, liver function tests, kidney function tests, urine examination were normal. Arterial blood gas, serum sodium, potassium, total proteins and albumin levels were in normal range. Serum calcium was $3.5 \mathrm{mg} / \mathrm{dl}$ (normal 9-11 mg/dl), Serum phosphorus was 6.1 $\mathrm{mg} / \mathrm{dl}$ (normal 1.5-3.0 mg/dl) EEG demonstrated generalized epileptiform activity. USG abdomen and pelvis was normal. CT brain demonstrated diffuse calcification of cerebral cortex, cerebellar cortex and basal ganglia. Para hormone assay level was $0.12 \mathrm{pmol} / \mathrm{l}$ (normal 1.6-6.9 pmol/1). Thyroid function tests were normal. Skeletal survey was unremarkable.

Patient received Calcium gluconate infusion $(10 \mathrm{ml}$ of $10 \%$ calcium gluconate at a rate of $1 \mathrm{ml} / \mathrm{min}$ ) for three days along with 1,25 cholicalciferol 1 microgram $/ 24$ hrs ( 0.01 0.10 micro gram $/ \mathrm{kg} / 24 \mathrm{hrs}$ ) along with carbamazepine 600 $\mathrm{mg} / 24$ hrs. 
Patient remained seizure free in hospital for nine days and was maintained on elemental calcium $1500 \mathrm{mg} / 24 \mathrm{hrs}$, Vitamin D3 one microgram/day. Serum calcium was maintained $6.9 \mathrm{mg} / \mathrm{dl}$ and serum phosphorus at $4.2 \mathrm{mg} / \mathrm{dl}$. Anti epileptics were continued and patient was urged to get his family for biochemical studies but he refused.

Patient lost follow up and treatment. He reported back after two months to our hospital in status epilepticus with serum calcium of $4.5 \mathrm{mg} / \mathrm{dl}$ and phosphorus $6.5 \mathrm{mg} / \mathrm{dl}$. After stabilization in ER, patient refused admission ${ }^{4}$

\section{Discussion}

Hypoparathyroidism can occur in hereditary setting presenting as Di george syndrome or vecardio facial syndrome typically having features of intellectual impairment, cataract and basal ganglia calcification. ${ }^{4}$

Idiopathic hypoparathyroidism is a rare disorder, which can be associated with auto immunity like vitiligo, moniliasis. It can coexist as polyglandular disorder. ${ }^{4}$

Pseudo hypoparathyroidism is end organ resistance to PTH, due to mutation of GS alfa protein coupled to PTH receptor. It presents as short stature, short metacarpals, subcutaneous calcifications and intellectual impairment. Variable degrees of resistance to TSH, LH and FSH are also present. $^{4}$

Pseudopseudo-hypoparathyroidism has phenotypic findings of pseudohypoparathyroidism without any abnormalities of calcium metabolism and runs in families.

Inappropriately high phosphorus and low calcium and deranged renal functions is seen in patients of CRF with PTH being ineffective. Hypomagnesaemia blunts effects of PTH with resultant hypocalcaemia and homeostasis is restored upon repletion of Magnesium and calcium replacement.

Acquired hypoparathyroidism occurs after exploratory thyroidectomy or parathyroidectomy. Radiation can induce hypoparathyroidism due to its immediate and delayed effects.

Deposition disorders like haemochromatosis, amyloidosis or sarcoidosis can present as acquired hypoparathyroidism but are difficult to diagnose and rectify.

In view of low calcium, high phosphorus, very low PTH, calcification on brain imaging and in presence of normal KFT, normal Magnesium the diagnosis of idiopathic hypoparathyroidism was established.

Such patients need to be managed with calcium infusion and activated Vitamin D3 during emergency presentations and maintained on calcium and vitamin D3 oral replacements thereafter for rest of their life. Patient should be regularly monitored for calcium levels which can go in hypercalcemic range, the therapy needs to be tailored. Role of antiepileptic drugs is not well established, but is usually co prescribed during status epilepticus or recurrent seizures, then progressively tapered.

\section{References}

1. Okazaki T, Takuwa Y, Yamamota M, et al. Ossification of the para vertebral ligament : a frequent complication of hypoparathyroidism. Metabolism 1984;33: 710-13.

2. Illum F, Dupont E. Prevalences of CT-detected calcification in the basal ganglia in idiopathic hypoparathyroidism and pseudohypoparathyroidism. Neuroradiology 1985;27: 32-37.

3. Singh Harigobal, Kumar N, Bhargava P. Fahr's syndrome as radiological quiz. Medical Journal Armed Forces India 1991;47:291-99.

4. Potts TJ. Diseases of parathyroid gland and other hyper and hypocalcemic disorders. Harrison's Principles of Internal Medicine 15th ed New York McGraw hill 2000 Vol 2, pp 2205-6.

5. Abe $\mathrm{S}$, Tojo $\mathrm{K}$, Ichida $\mathrm{K}$, et al. A rare case of idiopathic hypoparathyroidism with varied neurologic manifestations. Internal Medicine 1996;35:129-34. 